ON THE COAGULATION OF THE BLOOD OF SOME ARTHROPODS AND ON THE INFLUENCE OF PRESSURE AND TRACTION ON THE PROTOPLASM OF THE BLOOD CELLS OF ARTHROPODS.'

\title{
LEO LOEB.
}

(From the Biological Laboratory, Woods Holl, Mass., and from the J. H. L. Molson Laboratories of Pathology and Bacteriology, McGill University, Montreal.)

I. The Normal Coagulation of the Blood in Some Arthropods.

For this work the blood of Limulus polyphemus, Homarus Americamus, Platyonychus ocellatus, and some other arthropods, was used.

Coagulation of the blood takes place in a somewhat different way in Limulus, lobster and spider crab.

In Limulus the blood coagulates as a bulky mass which in the following 24 hours contracts considerably. A second coagulation of the fluid expressed from the clot may take place, to a slight extent, forming very loose gelatinous masses around the central clot, or more rarely, the whole serum may afterward coagulate.

In the lobster the first coagulum is usually relatively small, consisting of shreds; usually however, in the course of twenty minutes to an hour, the serum remaining after the first coagulation coagulates as a solid gelatinous mass which in the next twenty-four hours retracts but little. Very rarely no coagulation of the serum remains after the first coagulation takes place.

In the spider or lady-crab, the first coagulation is similar to that of the lobster blood. No second coagulation however takes place.

A combination of the following factors comes into play in producing the clotting of blood in arthropods.

1 This communication is in most parts only an abstract of a fuller report which shall appear later. It is intended to continue these experiments. 
I. The Agglutination of Blood Corpuscles.-This phenomenon may be observed uncomplicated by the other factors, $e . g$., if we collect blood in distilled water, in solutions of gelatine or of adrenalin. Under these conditions the cytoplasm of the cells itself furnishes a sticky substance necessary for agglutination. Coagulation of the blood of arthropods is partially based on the $a g$ glutination of the blood cells taking place spontaneously outside of the body.

2. Just as the cells agglutinate, so the cell protoplasm having left the ruptured cell body agglutinates, and the remaining part of the cell frequently has the appearance of still intact but smaller cells. The protoplasm of cells which have been absolutely dissolved may also agglutinate and form gelatinous masses.

3. About six to ten minutes after the shedding of the blood the blood cells, which in the meantime have been mostly collected in strings, begin to move in contact with solid bodies, $e . g$., the surface of the slide; the protoplasm of the cell becomes more and more drawn out, so that ultimately a fine network of flattened cells is formed in which for a certain time the anastomosing exoplasm can be distinguished from the granular endoplasm.

4. There also takes place the coagulation of a substance which previously was in solution. This coagulation is especially marked in the blood of the lobster, but it also takes place to a less degree in the blood of Limulus.

\section{On Some of the Factors Determining ine Second}

Coagulation of the Blood of the Lobster.

The conditions under which the coagulation of the substance first in solution in the blood of arthropods takes place can best be studied in the blood of the lobster. If, after the shedding of the blood, one quickly collects the first fibrin by shaking the vessel and then removing it with needles, the coagulation can be inhibited for longer or shorter periods, or even be entirely prevented. The fact that coagulation nevertheless usually takes place, although somewhat later, is partially to be explained by the fact that it is impossible to remove all shreds. These shreds mostly consist of agglutinated cells. If one examines the second gelatinous coagula formed at the bottom of the dish, even after 
having apparently most carefully removed the shreds, one frequently sees under the microscope a small mass of blood corpuscles which had not been removed from the blood, in the center of a small gelatinous coagulum.

In many cases, however, a second coagulation after having removed most of the shreds does not take place even if some of these shreds have remained. In the cases in which it does take place it is also possible that the cell protoplasm, derived from the solution of so many blood cells, may be sufficient to cause coagulation even if the absolutely intact blood cells themselves have been entirely removed. It may be found that by adding water to the blood during the first coagulation and afterwards filtering the diluted blood, the second coagulation can be inhibited with quite a certainty for a longer period than otherwise, or may be even entirely prevented. Dilutions of the blood therefore produce an absolute diminution in the formation of fibrin for a certain period, not only a relative one in proportion to the remaining fluid.

In the experiments made such diluted serum was afterwards distributed in small dishes, 4 or $5 \mathrm{cc}$. into each dish ; different substances were added to several of those dishes to determine the influence of these substances on coagulation.

It could be shown that the addition of about two or three pieces of shreds of fibrin derived from the first coagulation of the lobster blood had a very pronounced effect on the coagulation of the diluted serum. Without the addition of such fibrin coagulation either did not take place at all, or only after some hours or on the next day. The difference in the dishes with shreds of fibrin and those without the fibrin was very marked. The influence of these shreds derived from the first coagulation was a localized one, the clotting always beginning directly around the pieces of fibrin and from here advancing concentrically towards the periphery of the fluid.

The fibrin however produced by the first coagulation is not the only substance having such an effect; pieces of muscle of the lobster act about equally well. In these experiments the muscle was carefully washed so that all blood was removed. The fibrin of the blood of rabbits or rats, or muscle of frogs, was without 
effect. The fibrin of these latter animals was used as the fibrin of the lobster, or in some instances was for two hours put into lobster serum previous to the experiment, so that substances present in the fibrin which might inhibit coagulation were removed. Lobster fibrin, treated in this way, was active; the fibrin of rats and rabbits was without effect. If, however, to the lobster serum previously treated with fibrin of rabbits, fibrin of lobster was added, the serum coagulated; therefore, no substance inhibiting coagulation had been extracted from the mammalian fibrin.

Small pieces of the pancreas-liver of the lobster entirely prevented, as was to be expected, coagulation.

After pieces of the first lobster fibrin had been put for ten minutes or longer into absolute alcohol, then washed and dried, they lost almost entirely their power to produce coagulation. A trace of the second gelatinous coagulum, however, was sometimes still formed around such pieces. Control experiments showed that alcohol passing into the serum was not the cause of this loss of efficiency.

Pieces of fibrin put into chloroform water over night and even for three days, may still be very efficient, though not quite as active as fresh fibrin. Put into chloroform water for five days they lost to a large extent their efficiency. The addition of I cc. of chloroform water to 4 or $5 \mathrm{cc}$. of serum, or of a few drops of pure chloroform, to the serum had no more influence on the coagulation than the addition of an equal amount of water.

Heating of the fibrin in the water-bath to 46 or $47^{\circ}$ for 30 to 40 minutes, destroyed its efficiency to a great extent, though not completely. Heating up to $5 \mathrm{I}-54^{\circ}$ for 40 minutes, destroyed its efficiency entirely or almost so.

Heating of the serum to $46-50^{\circ}$ during 30 minutes, usually prevented the spontaneous coagulation of the serum. If, however, one adds to such serum previously heated, fresh fibrin, the serum coagulates almost as rapidly as normal serum.

The addition of I cc. of glycerine to $4 \mathrm{cc}$. of serum did not have any specific inhibiting influence. Also the addition of $\mathrm{I}$ cc. of a weak solution of pure gelatine did not have any specific influence. 
The addition of I cc. of water and 6-4 per cent. sodium chloride solution to the serum had in most cases but a slightly inhibiting effect.

In such solutions coagulation has occurred occasionally even more rapidly than with the pure serum ; an accidental admixture of a larger number of blood cells to this liquid may perhaps have been the cause. All these experiments, as well as most of the following ones, were made with the addition of shreds of fibrin obtained from the first coagulation of the lobster blood.

The addition of I cc. of a I per cent. solution of potassium cyanide or of a 16 per cent. solution of Witte's peptone, or of a saturated solution of urea, had a decidedly inhibiting effect. The peptone solution prevented the coagulation entirely; the potassium cyanide and urea solutions frequently had only an inhibiting action for some time, after perhaps i 6 hours (sometimes earlier) coagulation usually occurring. Generally only half an hour after the addition of these substances their effect became noticeable. The order in which these substances, according to their strength, acted on the serum, was the following: Potassium cyanide had the weakest and Witte's peptone the strongest action ; urea stood between these substances.

If, however, the experiments were made in this way, that the pieces of the first fibrin were in the beginning put for some time into potassium cyanide, urea or peptone solutions, then after having been washed out, put into dishes containing the blood serum, the order of the loss of efficiency of the fibrin was reversed. Pieces having been kept for one half hour to two hours in peptone solution, were almost as effective as fresh fibrin, even pieces having been kept in peptone over night were frequently still very active, although in many cases they had lost a considerable part of their efficiency. It is of importance that the peptone be well washed out for some hours, otherwise the peptone, becoming extracted and dissolved in the serum, prevents its coagulation.

Pieces having been kept for one half to two hours in a solution of urea and having been afterwards well washed, have lost the greatest part of their efficiency, and after having been kept for twelve hours have become absolutely ineffective. 
A I per cent. solution of potassium cyanide usually destroys the efficiency of the fibrin inside of half an hour. After having been kept for two hours in such a solution the fibrin becomes opaque.

One cubic centimeter of a 2 per cent. solution of potassium oxalate, or 5 drops of a ro per cent. solution of the same solution added to $4 \mathrm{cc}$. of serum, entirely prevents the coagulation.

One half cubic centimeter of a 2 per cent. solution of potassium oxalate inhibits the coagulation markedly.

The addition of 5 drops, or I cc., or 2 cc. of a 2 per cent. calcium chloride solution had, in one series of experiments, a slightly accelerating influence on the coagulation in comparison with control experiments which were made with the addition of I cc. of a weak sodium chloride solution. Calcium is probably present in a sufficient quantity in the serum and the farther addition of it can no longer exert a pronounced influence.

A more extended series of experiments on the action of different salts on the coagulation is to be made later. Some experiments have, however, already been made on the action of ammonium chloride (the marked action of urea suggested these experiments). This salt, compared with the corresponding solutions of sodium chloride, has a specifically inhibiting action on the coagulation. The addition of I cc. of a I 6 per cent. solution of sodium chloride frequently had a weak, though at times a somewhat stronger, inhibiting influence ; 7 drops of a 16 per cent. ammonium chloride solution, or I cc. of a 4 per cent. solution of the same solution, had a much more pronounced inhibiting influence. One cubic centimeter of a 16 per cent. solution of acetamid had but a slightly inhibiting effect. The addition of a few drops of ammonium prevented coagulation entirely; this, however, was probably merely an alkali-action.

If the mixtures of blood serum with the above substances are kept for 24 hours in a warm room, putrefaction of the liquid usually takes place. This putrefaction does not prevent coagulation although it may inhibit it somewhat.

After the second, gelatinous, coagulation has taken place, it is possible to separate a second serum from this coagulum. This second serum, to which fresh shreds of fibrin have been added, no longer coagulates. 
If, instead of the shreds of the first coagulation, the gelatinous coagulum of the second is added to the ordinary serum, efficiency of the latter fibrin is usually found not to be as pronounced as that of the first fibrin.

Foreign bodies, as such, added to the serum in place of fibrin or muscle, do not tend to accelerate coagulation. In no case could any marked influence be observed. Also platinum black was without any effect.

On the surface of the blood serum, kept in open dishes, usually a film is formed. This surface film in most cases radiates from the shreds of fibrin put into the serum. If we shake the serum for one half hour in dishes with uneven surfaces, macroscopically, visible membranes of fibrin are formed.

Extract of fibrin, which was previously kept in absolute alcohol, or of fresh fibrin under addition of chloroform water, have so far not shown an efficiency comparable to that obtained by the addition of fresh fibrin itself. One cubic centimeter of such an extract added to $4 \mathrm{cc}$. of serum, in most cases only caused a very insignificant acceleration of the coagulation in comparison to serum to which I cc. of a 6 per cent. sodium chloride solution had been added. Halliburton states that an extract of mammalian blood precipitated in alcohol and afterwards dried, produced coagulation in blood which had been collected in a magnesium sulphate soluion. I did not succeed in producing coagulation in this way; such a result would be contradictory to the one obtained by myself with the fibrin of rabbits and rats.

We may explain all these facts by the supposition that in the shreds produced by the first coagulation there is present a ferment or pro-ferment producing coagulation, and further, that such a ferment or pro-ferment must be present inside of the blood cells, because the first clot consists mainly of blood corpuscles and parts of these cells. Further, we are certain of the decidedly localized action of this ferment, as directly around the shreds of fibrin the second gelatinous fibrin is deposited. This can be explained by the assumption that the ferment can only slowly diffuse into the fluid; it must however nevertheless be able to diffuse through the gelatinous substance formed around the shreds, although as recent experiments seem to prove, even 
soap mixed with gelatine is unable to diffuse through the gelatine.

The local apposition of the gelatinous mass around the fibrin shreds produces a certain resemblance between the process of clotting and that of crystallization when caused by a crystal in a solution. If we make use of the hypothesis of the action of a ferment, we have to suppose, according to the results obtained by heating the serum, that also in the serum a small quantity of this ferment or pro-ferment is present ; further, that the ferment is partially destroyed by heating it up to $46-47^{\circ}$ C. and that it is almost entirely destroyed by heating it up to $5 \mathrm{O}-5 \mathrm{I}^{\circ} \mathrm{C}$. There is a quantitative relation present, the coagulation taking place the more rapidly the more fibrin is added to the serum. Although absolute alcohol is used to obtain mammalian fibrin ferment, and also, according to Halliburton, that of arthropods, in these experiments absolute alcohol destroyed efficiency of arthropod fibrin ferment in a very short time.

We saw that different kinds of cells and cell products of the same species of animals had the same accelerating effect on coagulation, that however the corresponding cells of other animals were without effect. The lack of specificity in the one case combined with the specificity with regard to the species of animals used, is similar to the specificity and lack of specificity respectively found in the precipitin reactions obtained after the injection of albuminous substances in different animals.

Delezenne found that contact with the muscle causes a coagulation of the blood of birds. If the contact with muscle is avoided, it is possible to keep the blood of birds liquid for a long time, although the white blood corpuscles are still present. Lobster blood, however, in contact with the blood corpuscles, does coagulate just as quickly as if in contact with muscle. These observations, notwithstanding the presence of some minor differences, seem to point out that in these phenomena we have to deal with facts of a general significance.

\section{The Conditions Under Which the First Coagulation Can be Inhibited or Entirely Prevented.}

The conditions under which the first coagulation can be prevented are different from those described above for the second 
coagulation. The following experiments were mainly made with adult Limuli :

(a) If one collects blood at once into saturated solutions of different salts, surpassing in quantity considerably the quantity of blood received, coagulation can be to a great extent inhibited or prevented. Magnesium sulphate, sodium chloride, potassium chloride, sodium nitrate, aluminium, ammonium sulphate, potassium oxalate, sodium citrate, potassium and sodium tartrate, potassium sulphate and sodium sulphate, all have a decidedly inhibiting effect, and to a less degree sodium carbonate.

If the solutions are diluted with one half or an equal amount of water, the effect shows a corresponding decrease.' Potass'im salts are less effective than sodium salts; the potassium nitrate is almost without effect; the oxalates do not have any markedly stronger effect than the other saturated salts ; it is therefore improbable that oxalates act in this case as precipitants of calcium, but rather as saturated salts. The blood corpuscles are in the main better preserved in these solutions. In the sodium and potassium sulphates they become roundish and send out processes after some time. In sodium nitrate and potassium and sodium chloride they retain on the whole an oval shape. Sabattani's hypothesis, that the inhibiting effect of salts is based on their action on calcium, preventing it from being ionized, becomes improbable, at least in this case. If the saturated solutions of the salts containing the blood are afterwards diluted with distilled water, many cell granules which had previously been preserved, now disappear. Many cells, however, seem to remain unchanged.

(b) Pyrrhol, resorcin and especially hydrochinon, have some inhibiting action on coagulation without preventing it entirely. Blood corpuscles remain more or less preserved in these solutions.

(c) Solutions of adrenalin chloride, $\mathrm{I}-300$, pilocarpine $\mathrm{I}-300$, and atropine $\mathrm{I}-300$, have a slightly inhibiting effect on coagulation, especially adrenalin. The cells here swell up and frequently arrange themselves through agglutination in epithelial-like order. Many cells, mainly at the margins where the adrenalin comes in contact with the blood, remain isolated or form only small clumps. The formation of a continuous coagulum however, at the margin does not take place frequently. 
(d) A 3 per cent. solution of formalin injected into lobsters or spider-crabs has, if the blood is shed soon afterwards, a more or less inhibiting effect on the coagulation, especially if the blood is then also collected in a 3 per cent. formalin solution.

Under such conditions it can be observed that all blood corpuscles of the lobster blood have granules, and that cells corresponding to lymphocytes are normally not present in the blood of the lobster. Their presence has lately been maintained. ${ }^{1}$

(e) The first coagulation of the blood can be prevented by previously heating the animals; the heat necessary to produce this is different in different species. The blood of small Limuli heated for $30-40$ minutes up to $50-54^{\circ}$ C. does not usually coagulate after having been shed. Lobster or Libinia, heated to $45-48^{\circ} \mathrm{C}$. for 30 minutes loses its coagulability. Correspondingly, lobsters and spider-crabs die at a lower temperature than small Limuli. The cells usually leave the body after the animal has been heated, in a round shape and later do not usually send out pseudopodia, or do so to a less degree only. The spreading out of blood corpuscles which usually takes place under normal conditions does not take place after previous heating of the animals, or if the animal has been injected with formalin.

(f) Blood collected in the liver-pancreas juice does not coagulate to the same extent as ordinary blood. A large number of the blood corpuscles are usually rapidly changed into granules and many of them soon become entirely dissolved through the action of the proteolytic ferment.

$(g)$ By previous injections of Limulus blood into turtles I have not succeeded in obtaining either precipitins for Limulus blood or substances which inhibit the activity of its fibrin ferment.

(h) Limulus blood collected in gelatine solutions of different concentration did not coagulate as readily as the blood collected in water, gelatine having a decidedly inhibiting action. If the solution of gelatine was very weak care had to be taken to avoid the shaking of the blood, otherwise coagulation took place. The blood corpuscles in gelatine were swollen and frequently formed epithelial-like rows.

${ }^{1}$ A fuller description of the blood corpuscles of Limulus is given in a previous paper: Journal of Medical Research, Vol. II., January, 1902. 
(i) Collected in glycerine or in chloroform the blood coagulates.

$(j)$ The blood of the lobster collected in saturated urea solutions or in a 25 per cent. peptone solution, forms a gelatinous mass which does not contract in the usual way. Urea solutions have a strongly dissolving influence on the blood corpuscles. The shreds resulting from the first coagulation of the lobster blood put into urea solutions, become transparent and swell ; in glycerine they also become transparent but do not swell; returned into water they regain their usual characters.

\section{On the Influence of Mechanical Agencies on the Protoplasm of the Blood Celies of Some Arthropods.}

It has been observed by previous investigators that the blood cells may send out long processes which can adhere to solid particles. It is however possible to produce a much greater change. The protoplasm of a little clump of blood cells can be transformed into a system of threads of different sizes. It is best to use for such experiments cells which have been well preserved and are somewhat swollen; it is also necessary to use blood in which it is possible to exclude the presence of an extra-cellular mass of fibrin. Such cells are found, e. g., in blood collected in gelatine solutions or in adrenalin or, also, in hydrochinon.

The necessary traction can be applied either by putting a second slide on the first containing the blood in solution and separating the two slides after they have been pressed together or by whipping with a needle the blood cells on the slide. The whole process can be followed under the microscope; one can see how cells arranged in a row become transformed into a system of threads which no longer indicates that it is derived from cells. Sometimes however we can see in the center of such threads the nuclei of the cells, adapting themselves to the thickness of the thread, or they may bulge out of the center of the fiber as little spheres. Under such circumstances one single cell can be changed into a very long thread. It can also be seen that frequently spindle-shaped cells arrange themselves into 
a single row and may form one long continuous thread passing over a large part of the slide.

Sometimes very little effort is needed to achieve such a transformation. If we move the solution containing a few drops of blood in one direction the flow of the liquid may be sufficient to arrange the cells into rows and draw them out into continuous, long threads.

Such fibrils are, however, not only formed from the protoplasm of the entire cell but they also may be formed inside of cells. This can be observed, for instance, if we exert traction by a needle in a network of cells spread out on the slide, $e . g$., about half an hour after the blood has been shed. If we merely exert pressure on masses of cells we may change them into a structureless gelatinous mass. By exerting afterwards traction on such a gelatinous mass, using two slides, we can change this mass into a system of fibrils of different diameters; such fibrils can again be rolled up into a gelatinous mass. The protoplasm has in this case the consistency of a dough, differing however in that it is somewhat more elastic.

If we apply these mechanical agencies to cells immediately after they have left the body, we find the protoplasm more brittle and the cells can under these conditions by pressure easily be separated into small granular particles.

A transformation into fibrils can also be produced from the protoplasm which had previously left the body of the ruptured cell, or from the protoplasm of exploded cells. The same agencies, traction and pressure, are also able to make the granules of the cells disappear. Cells which had been previously kept for a longer period in saturated salt solutions or in formalin, no longer yielded such a fibrillar mass.

The physical properties of this fibrin-like mass are very similar to the fibrin formed through the coagulation of the extra-cellular part of the blood. The cellular fibrin can be drawn out, and as it is somewhat elastic, may afterwards slowly retract. It is also sticky. The cells themselves have similar physical properties. If they are spread out, as is usually the case, one half to one hour after coagulation they are kept under great tension, and if liberated from the surface of the slide by a needle they retract. 
They also are sticky. A cell, hanging on a fiber after coagulation of the blood has taken place, sticks to the surface of the slide; after having sent out processes, if as a result of outside motion a pseudopodium of the cell touches the surface of the glass.

Very similar fibrils can be produced if we subject the blood serum of Limulus or lobster to the same mechanical agencies. It usually however needs more exertion to obtain the same result from these colloidal solutions than from the cells.

\section{V.}

Certain analogies may be pointed out which exist between certain processes during coagulation of the blood and certain other phenomena.

(a) Inside of the body blood corpuscles have an oval shape and do not send out pseudopodia. After having left the body certain changes in the environment take place, the blood cells send out pseudopodia, and somewhat later they begin to spread out over the whole surface of the slide, forming one continuous network. We see a certain analogy between this process and the emigration of leucocytes under so-called inflammatory conditions.

The changes which lead to the spreading out of the blood corpuscles of arthropods cannot, under the given conditions, be determined by chemotropic influences acting from certain directions. The chemical conditions on the slide are the same on, all sides ; nevertheless, the cells move and spread out. Inflammatory conditions in the higher animals also mean a change of the normal environment brought by toxic or other causes. Thus it may be that the leucocytes of vertebrates do not migrate primarily under the influence of chemotactically acting substances, but under the influence of certain physico-chemical factors which bring about changes in the state of the protoplasm of these cells; direct chemical stimulants may be added secondarily.

(b) During regenerative processes of the epithelium movements of the cells take place independent of preceding cell multiplication. The epithelial cells under these conditions usually move in contact with solid bodies, parts of which, as I have previously shown, may be taken into the cell body itself. It can be observed under 
these conditions that the protoplasm of the epithelium is in very close connection with the solid body, for instance the coagulum forming a scab, and if one raises the scab from the wound parts of the epithelium may remain in contact with it and are raised with it. It is not unlikely that similar changes in the consistency of the protoplasm of the epithelial cells take place during regeneration, as take place in the blood cells after they have left the body, and the adhesion may in both cases have to be explained by a change in the consistency of the protoplasm which makes the cell or part of it sticky.

(c) We have been able to show that under the influence of traction the protoplasm of the cells assumes a fibrillar structure and that these fibrils frequently are continuous through a number of cells. Similar phenomena can be observed in many other cells. Epithelial fibrils, for instance, are not limited to one cell and especially under the influence of traction exerted during the regenerative process, the fibrils passing through more than one cell are drawn in the direction of the traction exerted and indicate therefore the movements of the epithelium. In a similar way we find fibrils passing through many cells, for instance in the heart muscle, in the developing white fibrous tissue and neuroglia and probably also in the peripheral nerves and in ganglia cells. The same mechanical factor bringing about fibrillation which can be observed experimentally in the protoplasm of blood cells, may be of importance also in these cases. The main factor is of course the specific structure of the different cells determining the specific structure of these fibrils.

(d) The blood corpuscles which in the body under normal conditions do not change their oval form, produce outside the body an agglomeration of cells which may be compared to a tissue. Under certain conditions, $\ell . g$., in gelatine or in adrenalin, or even in distilled water, many cells form through agglutination an epithelial-like tissue; under ordinary conditions, however the cells soon form intercellular substances and the appearance of connective tissue is produced. The network of spread-out cells closely resembles the reticular tissue. The exoplasm of the spread-out cells under the influence of slight mechanical agitation, takes on a fibrillar character. If we make sections through a coagulum in 
which the blood corpuscles have spread out, the appearance of connective tissue is presented. We see apparently intact cells surrounded by fibrils. These cells, however, only represent the endoplasmodic part of the original blood corpuscles, the exoplasmatic part having been transformed into fibrils. Many of these fibrils stain very well by Mallory's connective tissue stain.

In a similar way, the fibers of fibrous tissue and of neuroglia in mammalians seem to be produced from the exoplasm of the cells. In coagulating blood, however, whole cells may be changed into fibers. Here protoplasm having left the cell body and being now entirely intercellular may also form fibrils. Such an occurrence can easily be observed under the microscope in the blood, these changes taking place in a short time. It would be more difficult to prove such an occurrence in the development of connective tissue. The morphological similarity (not identity) between the processes in the blood cells and connective-tissue cells being apparently so great, it ought to be considered, whether an intercellular origin of fibers from protoplasm, secondarily disconnected from cells may not also take place in connective tissue.

We can observe that the granules of the blood cells frequently spread out and assume in the end an intercellular situation, and that they not infrequently become included in fibrils. Similar observations have been made in the developing cartilage for instance.

As we have seen, the formation of intercellular substances in the coagulating blood can be prevented if we collect the blood cells in certain solutions, which inhibit that further changes in the cells take place without preventing the possibility of such changes, if the solution is changed afterwards. We may therefore say that one of the conditions under which the formation of an intercellular substance takes place is the presence of a certain fluid which surrounds the blood cells which have left the body. The serum has therefore a more or less cytolytic power and the formation of "connective tissue" is based on a partial destruction or dissolution (cytolysis) of the blood cells by which protoplasm leaving the cells becomes changed into intercellular fibrils.

\section{Summary.}

I. The following factors play a part in the coagulation of the blood of arthropods : 
(a) Agglutination of blood cells.

(b) The formation of a gelatinous mass, and secondarily the formation of fibrils from protoplasm exuded from cells and from protoplasm of cells entirely dissolved.

(c) The spreading out of the blood cells during the next few hours after shedding of the blood.

(d) The coagulation of a fibrinogenous substance.

2. The agglomeration of the blood cells leads to the formation of tissue-like structures. The formation of the fibrillar intercellular substance corresponds to the formation of fibrillar connective tissue. By changing the cytolytic medium in which the cells are suspended, the formation of an intercellular fibrillar substance can be prevented. By agglutination of cells which remain well preserved, an epithelial arrangement of the blood cells can be brought about.

3. Inside of the normal body the blood cells do not change their form ; this however takes place outside of the body, without the presence of certain specifically directing chemotropic stimuli. The cell protoplasm moves in contact with the solid surface to which it sticks. In a similar way the leucocytes react during an inflammatory process and a certain analogy also exists between this process and the movement of the epithelium during regeneration.

4. Coagulation of the blood can be prevented or inhibited by receiving it in different solutions, such solutions usually having also a preservative influence upon the cells. Oxalates act in a similar way to other salt solutions, that is if they are very concentrated. This points to the fact that in all probability the precipitation of calcium is, under these conditions, not the essential factor. Sodium and potassium sulphate have, in a similar way as sodium nitrate and sodium and potassium chloride, an inhibiting influence upon coagulation. The blood cells themselves, however, are influenced in a different way by the two sulphates and the other salts.

5. The coagulation of the blood is inhibited by receiving the blood in gelatine solutions; collecting it in oil is without influence.

6. The second coagulation of the lobster blood is inhibited by means different from those acting on the first coagulation. The 
facts observed during the second coagulation can be explained through the hypothesis of the action of a fibrin ferment present in the blood cells and in the muscle of the lobster, which however is not present in the blood cells or in the muscle of certain vertebrates. This points to a multiplicity of fibrin ferments in different species of animals and points to the identity of the ferment in different tissues of the same species of animals. This specificity in the one case and the want of it in the other case corresponds to a similar specificity and want of specificity as has been found to exist with the precipitins obtained through injection of certain body fluids or albuminous substances into different species of animals.

7. Potassium cyanide, urea and solutions of peptone inhibit the second coagulation. If these substances are add to the serum their inhibiting influence is in a reverse proportion to thire strength if they act directly upon the fibrin which contains the ferment. Potassium cyanide has the strongest, peptone the weakest action upon the fibrin, and conversely, peptone has the strongest and potassium cyanide the weakest action on being mixed with serum.

8. The necessity of the presence of calcium for the second coagulation can be easily proved. Ammonium chloride has a stronger inhibiting action on the second coagulation than sodium chloride.

9. Mechanical agencies, namely, pressure and traction, change the cell protoplasm into a system of fibers which cannot be distinguished in its physical properties and appearances from the extra-cellular fibers. The cell granules disappear under the influence of traction and pressure, just as the granules disappear spontaneously in the cells during coagulation of the blood. By traction and pressure exerted upon the blood serum free from cells, similar fibrillar structures can be produced as in the protoplasm of the cell. The fibrillar structure of the protoplasm seems therefore to be a secondary condition, produced by mechanical influence upon albuminous substances which may be common to the protoplasm of the cell and to solutions of certain albuminous substances.

IO. By pressure and traction the cell protoplasm can be changed into fibers whose arrangement is determined by the 
direction in which the mechanical forces act ; mechanical factors may also determine the direction of the fibrils in connective tissue and bone.

II. The significance of the blood corpuscles for the coagulation of the blood consists therefore in the following:(i) The cells themselves are transformed into substances similar to fibrin, (ii) They accelerate a coagulation taking place in the surrounding fluid ; some facts point to the possibility that the coagulating substance contained in the serum has its origin in the blood cells.

I2. Towards foreign substances introduced into the body of the animal, the blood corpuscles of Limulus do not behave as actively as the blood corpuscles of mammals. An active penetration into these foreign substances cannot be observed in Limulus, the cells only take part in the formation of the coagulum around the foreign substance. 


\section{$2 \mathrm{BHL}$ Biodiversity Heritage Library}

Loeb, Leo. 1903. "ON THE COAGULATION OF THE BLOOD OF SOME ARTHROPODS AND ON THE INFLUENCE OF PRESSURE AND TRACTION ON THE PROTOPLASM OF THE BLOOD CELLS OF ARTHROPODS." The Biological bulletin 4, 301-318. https://doi.org/10.2307/1535851.

View This Item Online: https://www.biodiversitylibrary.org/item/16879

DOI: https://doi.org/10.2307/1535851

Permalink: https://www.biodiversitylibrary.org/partpdf/34059

\section{Holding Institution}

MBLWHOI Library

\section{Sponsored by}

MBLWHOI Library

\section{Copyright \& Reuse}

Copyright Status: NOT_IN_COPYRIGHT

This document was created from content at the Biodiversity Heritage Library, the world's largest open access digital library for biodiversity literature and archives. Visit BHL at https://www.biodiversitylibrary.org. 